\title{
Brain Regions Related to Tool Use and Action Knowledge Reflect Nicotine Dependence
}

\author{
Yavor Yalachkov*, Jochen Kaiser, and Marcus J. Naumer* \\ Institute of Medical Psychology, Goethe-University, D-60528 Frankfurt am Main, Germany
}

In addition to reward- and craving-related processes, habitual mechanisms play an important role in addiction. While the dorsal striatum has been proposed to code for the motivational state of habitual drug-seeking actions, the neural underpinnings of the corresponding drug-taking skills and action knowledge remain poorly understood. We used functional magnetic resonance imaging (fMRI) and a behavioral orientation affordance paradigm to investigate the neural and behavioral correlates of automatized drug-taking actions in nicotine dependence. Smokers exhibited higher fMRI activations than nonsmokers when viewing smoking-related but not when viewing control images. These group differences in fMRI activations were located not only in brain regions associated with craving and habitual learning (left ventral and dorsal striatum, dorsolateral prefrontal cortex, insula, uncus, medial frontal gyrus, right subcallosal gyrus, and bilateral parahippocampal gyrus), but also in a network of brain regions which has been strongly implicated in the encoding of action knowledge and tool use skills (bilateral premotor cortex, left superior parietal lobule, and right lateral cerebellum). A behavioral affordance reaction-time task indicated that smokers, but not nonsmokers, showed an automatized responsiveness to smoking paraphernalia similar to everyday objects. Moreover, smokers showed strong intercorrelations between fMRI activations in tool use-related brain regions, behavioral responsiveness to smoking-related cues, and severity of nicotine dependence. Apparently smoking-related action representations in smokers are stored in brain regions typically representing tool use skills and action knowledge. Most importantly, cortical and behavioral correlates of the respective drug-taking skills vary with the individual degree of nicotine dependence.

\section{Introduction}

With an estimated death toll of $>5$ million per year (World Health Organization, 2008) nicotine dependence is the addiction with the strongest impact on public health. While sophisticated medical and psychotherapeutic treatments have contributed to a certain increase in abstinence rates, even the most effective approaches have long-term success rates of only $\sim 30 \%$ (Piasecki, 2006). While research has focused mainly on the reward system and craving, there is evidence that relapse into drug-taking behavior is not always related to conscious craving (Miller and Gold, 1994). Instead, the high change resistance of smoking behavior might be attributable also to deeply embedded habitual processes, which are compulsive and almost automatic. From a cognitive point of view, these habitual mechanisms are encoded as automatized action schemata, which develop with repeated use and contain information underlying the efficient initiation and execution of drug-taking behavior (Tiffany, 1990). These schemata can be triggered rapidly by smoking cues, thus increasing the probability that smokers will perform substance-use actions

\footnotetext{
Received 0ct. 10, 2008; revised March 13, 2009; accepted March 15, 2009.

We thank David Gilbert, Sandra Anti, Oliver Döhrmann, Christian Altmann, and Iver Iversen for their support and assistance.

*Y.Y. and M.J.N. contributed equally to this work.

The authors declare no competing financial interests.

This article is freely available online through the J Neurosci Open Choice option.

Correspondence should be addressed to Yavor Yalachkov, Institute of Medical Psychology, Goethe-University,

Heinrich-Hoffmann-Strasse 10, D-60528 Frankfurt am Main, Germany. E-mail: yalachkoø@med.uni-frankfurt.de. DOI:10.1523/JNEUROSCI.4891-08.2009

Copyright $\odot 2009$ Society for Neuroscience $\quad 0270-6474 / 09 / 294922-08 \$ 15.00 / 0$
}

independently of intentional processes. It has been shown that smoking behavior becomes automatized in frequent smokers (Baxter and Hinson, 2001; Field et al., 2006). Furthermore, recent synaptic plasticity research has identified the dorsal striatum as the region contributing to the establishment of persistent drugrelated habits (Gerdeman et al., 2003). While the activation patterns in the dorsal striatum reflect the formation of powerful stimulus-response associations (Everitt and Robbins, 2005), very little is known about the neural substrates of the corresponding drug-taking skills and action knowledge.

The present study explored the neural and behavioral correlates of drug-taking skills and smoking-related action knowledge in smokers and their associations with the individual degree of nicotine dependence. Smokers and nonsmokers were scanned with functional magnetic resonance imaging (fMRI) while viewing smoking-related and control images. We hypothesized that smoking-related but not control cues trigger the respective skill and action representations, which should be reflected by higher fMRI activations in smokers compared with nonsmokers in brain regions known to encode tool use skills and action knowledge. Furthermore, as automatized behavioral patterns are executed relatively fast and efficiently once they are triggered (Tiffany, 1990), we expected an increased behavioral responsiveness to smoking-related objects in smokers compared with nonsmokers. To assess behavioral responsiveness to both everyday and smoking-related objects we used an orientation affordance paradigm. This is a stimulus-response compatibility paradigm which has been used in numerous studies (Tucker and Ellis, 1998; Symes et al., 2007; Vainio et al., 2007; Riggio et al., 2008) showing 
that certain action-related properties of a stimulus (e.g., spatial information in terms of left/right orientation) generate automatic response codes (Tucker and Ellis, 1998). Thus, it is a suitable instrument to study the automatic activation of motor representations by graspable tools (Tucker and Ellis, 1998; Vainio et al., 2007). Since the automatization of drug-use behaviors increases with repeated practice, action representations on both the neural and behavioral levels should correlate with the individual degree of nicotine dependence.

\section{Materials and Methods \\ fMRI study}

Subjects. All participants were right handed, had normal or corrected-tonormal vision and were students or university academic staff. The smokers $(n=15$, mean age 27.1 years $(\mathrm{SD}=3.8), 6$ men and 9 women) were instructed to smoke according to their usual habits. Their individual level of nicotine dependence was evaluated with the Fagerström Test for Nicotine Dependence (FTND) (Bleich et al., 2002). The mean FTND score accounted to $3.13, \mathrm{SD}=2.39$. We recruited light as well as heavy smokers to represent the whole spectrum of smoking behavior. The nonsmokers [ $n=15$, mean age 28.7 years ( $\mathrm{SD}=6.8), 6$ men and 9 women] claimed that they had never smoked. After complete description of the study to the subjects, written informed consent was obtained.

Experimental design. During the experiment a total of 40 grayscale images from different categories were presented to the participants. The first category comprised images of humans smoking cigarettes ("smoke" condition) and the second category consisted of images of humans holding pens or glasses in their hands and mouths ("control" condition). The other three categories (data not reported here) consisted of images of everyday tools and animals and scrambled images. The images of humans were taken from the International Smoking Image Series (Gilbert and Rabinovich, 2006). We ensured that all images had the same size.

The participants underwent four functional runs and one anatomical scan. A dummy block at the beginning of each run ensured that T1 saturation effects were avoided. The eight images from the five categories were presented in separate blocks. Blocks for each image category were repeated three times within each run in pseudorandomized order, thus resulting in 15 experimental +1 dummy block per run. Blocks lasted for $16 \mathrm{~s}$ and alternated with fixation intervals of equal length. Images were presented in a randomized order for $1500 \mathrm{~ms}$ each with $500 \mathrm{~ms}$ interstimulus intervals in a block. We chose a passive paradigm to avoid confounding task-related sensorimotor activations. The participants were instructed to fixate the white cross and attend the images, which were projected onto a vertical screen positioned inside the scanner. Subjects viewed the screen through a mirror.

The fMRI procedure was performed on a 3-tesla Magnetom Allegra scanner (Siemens) at the Brain Imaging Center in Frankfurt am Main. A gradient-recalled echo-planar imaging sequence with the following parameters was used: 34 slices; repetition time (TR) $2000 \mathrm{~ms}$; echo time (TE) $30 \mathrm{~ms}$; field of view $192 \mathrm{~mm}$; in-plane resolution $3.3 \mathrm{~mm}^{2}$; slice thickness $3 \mathrm{~mm}$; gap thickness $0.3 \mathrm{~mm} ; 264$ volumes per functional run. For the anatomical imaging a magnetization-prepared rapid-acquisition gradient echo sequence (TR $2300 \mathrm{~ms}$, TE $3.49 \mathrm{~ms}$, flip angle $12^{\circ}$, matrix $256 \times 256$, and voxel size $1.0 \times 1.0 \times 1.0 \mathrm{~mm}^{3}$ ) was used.

Statistical analysis. Data were analyzed using the BrainVoyager QX 1.10.4 software package (Brain Innovation). Preprocessing included the following steps: three-dimensional motion correction, linear-trend removal and temporal high-pass filtering at $0.0054 \mathrm{~Hz}$, and slice-scan-time correction with sinc interpolation. Functional and Talairachstandardized anatomical data were used to build a voxel time course file and were spatially smoothed with a Gaussian filter of $8 \mathrm{~mm}$ full width at half maximum. Volume-based statistical analyses were performed at the whole-brain level. For each voxel, the time course was regressed on a set of dummy-coded predictors representing the experimental conditions. To account for the shape and delay of the hemodynamic response, the predictor time courses (boxcar functions) were convolved with a gamma function.

First, a two-way random-effects analysis (RFX) ANOVA with factor 1 "stimulus category" (smoke vs control) and factor 2 "group" (smokers vs nonsmokers) was calculated ( $n=30$ [15 smokers, 15 nonsmokers]). Then, a whole-brain statistical map for the interaction "stimulus category" X"group" was computed. Thus, we were able to show which regions exhibited differential fMRI activations across groups and object categories. Further analysis of these regions was performed with a ROIbased random-effects general linear model to identify the group/object differences which had caused the significant RFX ANOVA interaction. Unpaired $t$ tests of the estimated $\beta$ values for the two groups in the smoke and control conditions were performed to reveal the regions in which the fMRI activation during presentations of smoking-related objects was higher in smokers than nonsmokers.

For the analysis of correlations between fMRI cue reactivity and the individual degree of nicotine dependence a whole-brain RFX ANCOVA with the contrast "smoke > control" and the covariate "FTND scores" was computed ( $n=15$ smokers, "RFX ANCOVA-FTND"). The resulting statistical map showed the regions where the $\beta$ weights of the GLMcontrast "smoke $>$ control" and the individual FTND scores correlated significantly.

A similar procedure was used for correlations between fMRI cue reactivity and the behavioral task performance (see below). A whole-brain RFX ANCOVA with the contrast "smoke $>$ control" and the covariate "affordance index for smoking objects" was computed ( $n=8$ smokers; "RFX ANCOVA-affordance"). The resulting statistical map showed the regions where the $\beta$ weights of the GLM-contrast "smoke > control" and the individual affordance indices for smoking objects correlated significantly.

The RFX ANCOVA-FTND was computed with the data of 15 participants who passively viewed smoking-associated and control images. The RFX ANCOVA-affordance was based on the data of 8 smokers who were involved additionally in the active stimulus-response compatibility task. According to our hypotheses, automatized smoking-related skills and action knowledge should be activated in both cases and may share common neural correlates. However, due to the different experimental designs, we did not expect that the two statistical maps resulting from the RFX ANCOVA-FTND and RFX ANCOVA-affordance would reveal identical regions. We performed a further ROI-based analysis of the two statistical whole-brain maps to assess the similarity of the neural correlates of automatized smoking-related schemata in the two tasks. In the regions revealed by the whole-brain RFX ANCOVA-FTND, a ROI-based RFX ANCOVA with the contrast "smoke $>$ control" and the covariate "affordance index for smoking objects" was performed. Similarly, a ROIbased RFX ANCOVA with the contrast "smoke > control" and the covariate "FTND" was computed in the regions revealed by the wholebrain RFX ANCOVA-affordance. All statistical maps were corrected for multiple comparisons using a cluster-size threshold procedure and projected onto anatomical data sets averaged across all 30 participants.

\section{Behavioral study}

Subjects. We recruited 8 of the smokers [mean age 27.5 years $(S D=6.09)$, 3 men and 5 women; mean FTND score 2.38 (SD = 2.67)], who had participated in the fMRI experiment and whose FTND scores had remained stable over the time interval between the fMRI and behavioral experiments (on average 5 months), and 8 nonsmokers [mean age 28.13 years $(\mathrm{SD}=3.27), 3$ men and 5 women]. All the participants were righthanded students or university academic staff and had normal or corrected-to-normal vision. The smokers were instructed to smoke according to their usual habit. After complete description of the study to the subjects, written informed consent was obtained.

Experimental design. The participants sat in a dark, soundproofed room in front of a monitor on which visual stimuli were displayed. The experiment comprised five runs with 112 trials each. Each trial began with a white fixation cross visible for $1500 \mathrm{~ms}$. Afterwards, a randomly chosen object was presented for $700 \mathrm{~ms}$. Then the white fixation cross appeared again over the object and after $150 \mathrm{~ms}$ changed its color randomly from white to blue or brown. The new color was kept for $180 \mathrm{~ms}$ and then the fixation cross turned its color back to white. Half of the participants was instructed to respond with the left control button on the keyboard to a color change from white to blue and with the right control 
Table 1. Brain regions showing significant whole-brain RFX ANOVA interaction "stimulus category $\times$ group" (df $=28, p<0.001$, corrected for multiple comparisons) and ROI-based unpaired, two-tailed $t$ tests ( $\mathrm{df}=28)$ for group differences between responses to stimuli from the "smoke" versus "control" categories

\begin{tabular}{|c|c|c|c|c|c|c|c|c|c|}
\hline \multirow[b]{2}{*}{ Region } & \multicolumn{3}{|c|}{ Talairach coordinates } & \multirow[b]{2}{*}{ No. of voxels } & \multirow[b]{2}{*}{ Fvalue } & \multicolumn{2}{|c|}{ Smoke stimuli ${ }^{a}$} & \multicolumn{2}{|c|}{ Control stimuli $^{a}$} \\
\hline & $x$ & $y$ & $z$ & & & $t$ value & $p$ & $t$ value & $p$ \\
\hline Left ventral/dorsal striatum & -11 & 10 & -2 & 3069 & 8.23 & 4.838 & $0.000 * *$ & -1.133 & $>0.05$, n.s. \\
\hline Left insula & -36 & 13 & 9 & 1485 & 7.84 & 3.856 & $0.001^{* *}$ & -0.975 & $>0.05$, n.s. \\
\hline Left dorsolateral prefrontal cortex & -39 & 30 & 31 & 2690 & 10.14 & 3.336 & $0.002^{* *}$ & -1.709 & $>0.05$, n.s. \\
\hline Left medial frontal gyrus & -19 & 9 & 43 & 514 & 6.76 & 2.925 & $0.007^{* *}$ & -1.906 & $>0.05$, n.s. \\
\hline Left premotor cortex & -15 & -12 & 59 & 1066 & 7.84 & 2.879 & $0.008^{* *}$ & -1.781 & $>0.05$, n.s. \\
\hline Right cerebellum & 33 & -48 & -28 & 1259 & 7.64 & 2.837 & $0.008^{* *}$ & -0.123 & $>0.05$, n.s. \\
\hline Left uncus & -31 & 2 & -19 & 706 & 8.61 & 2.766 & $0.01^{*}$ & -2.842 & $0.008^{* *}$ \\
\hline Left parahippocampal gyrus & -13 & -28 & -7 & 973 & 7.68 & 2.742 & $0.011^{*}$ & -2.461 & $0.02^{*}$ \\
\hline Right subcallosal gyrus, Brodmann area 34 & 28 & 4 & -12 & 1866 & 8.53 & 2.522 & $0.018^{*}$ & -1.724 & $>0.05$, n.s. \\
\hline Left superior parietal lobule & -24 & -61 & 46 & 2050 & 8.28 & 2.496 & $0.019^{*}$ & -1.029 & $>0.05$, n.s. \\
\hline Right parahippocampal gyrus & 32 & -28 & -20 & 1594 & 7.67 & 2.468 & $0.02^{*}$ & -1.915 & $>0.05$, n.s. \\
\hline Right premotor cortex & 31 & 12 & 38 & 2117 & 7.58 & 2.123 & $0.043^{*}$ & -2.146 & $0.041^{*}$ \\
\hline Left cerebellum & -35 & -39 & -30 & 435 & 7.83 & 1.700 & $>0.05$, n.s. & -2.243 & $0.035^{*}$ \\
\hline Right posterior middle temporal gyrus & 50 & -61 & 6 & 1057 & 8.22 & 1.377 & $>0.05$, n.s. & -1.069 & $>0.05$, n.s. \\
\hline Left posterior middle temporal gyrus & -38 & -75 & 11 & 656 & 7.19 & 1.007 & $>0.05$, n.s. & -1.842 & $>0.05$, n.s. \\
\hline Right posterior cingulate, BA 30 & 24 & -64 & 8 & 879 & 8.64 & 0.824 & $>0.05$, n.s. & -3.484 & $0.002^{* *}$ \\
\hline Right middle temporal gyrus/occipital lobe & 42 & -70 & 14 & 791 & 7.39 & 0.242 & $>0.05$, n.s. & -2.052 & $0.05^{*}$ \\
\hline
\end{tabular}

${ }^{a}$ Positive $t$ values indicate higher $\beta$ values for the smokers than for the nonsmokers. Negative $t$ values indicate higher $\beta$ values for nonsmokers than for smokers. ${ }^{*} p<0.05 ;{ }^{* *} p<0.01 ;$ n.s., not significant.

button to a color change from white to brown. The other half of the participants received the inversed mapping instruction.

Grayscale images of objects from two categories were used: (1) everyday tools/manipulable objects (e.g., cup, scissors, hammer, etc.) and (2) smoking-related objects (e.g., cigarette, lighter, etc.). Images originated from commercially available image collections (150.000 PhotoObjects Graphics Collection and The Big Box of Art 615 000, Hemera Technologies). We ensured that all images had the same size. Each of the objects was presented with its handle or side where the object is usually grasped in left or right orientation. Thus, we had a $2 \times 2 \times 2 \times 2$ experimental design (within-subjects factors: object category, responding hand, object orientation; between-subjects factor: smokers vs nonsmokers). Seventy trials were presented for each of the 8 within-subjects conditions.

Statistical analysis. Reaction times and error rates were recorded for each participant and mean reaction time was calculated for each experimental condition. Following a well established statistical analysis procedure (Tucker and Ellis, 1998; Vainio et al., 2007), trials with reaction times deviating by $>2$ SDs from the participant's mean were excluded from the analysis [mean eliminated trials 23.6 (4.2\% of all 560 trials per subject)].

The orientation affordance effect has been defined as a shorter reaction time when the side of the response hand and the orientation of the presented prime object are identical (e.g., a response with the left hand to an object oriented to the left) compared with when they differ (e.g., a response with the left hand to an object oriented to the right) (Tucker and Ellis, 1998; Vainio et al., 2007). To facilitate the search for more specific interactions (e.g., group $\times$ object category interaction for the affordance effect), we computed an "affordance index" (AI) separately for each object category across both hands according to the following formula:

$$
\mathrm{AI}=\left[\frac{\left(\mathrm{LH}_{\mathrm{RO}}-\mathrm{LH}_{\mathrm{LO}}\right)}{\left(\mathrm{LH}_{\mathrm{RO}}+\mathrm{LH}_{\mathrm{LO}}\right.}+\frac{\left(\mathrm{RH}_{\mathrm{LO}}-\mathrm{RH}_{\mathrm{RO}}\right)}{\left(\mathrm{RH}_{\mathrm{LO}}+\mathrm{RH}_{\mathrm{RO}}\right)}\right] / 2,
$$

where $\mathrm{LH}_{\mathrm{RO}}$ is the mean reaction time for the left hand and rightoriented objects; $\mathrm{LH}_{\mathrm{LO}}$ is the mean reaction time for the left hand and left-oriented objects; $\mathrm{RH}_{\mathrm{LO}}$ is the mean reaction time for the right hand and left-oriented objects; and $\mathrm{RH}_{\mathrm{RO}}$ is the mean reaction time for the right hand and right-oriented objects. Thus, a higher affordance index for an object category indicates shorter reaction times for congruent combination between responding hand and orientation of the respective object (i.e., left hand and left object orientation, right hand and right object orientation) compared with an incongruent combination of the same (i.e., left hand and right object orientation, right hand and left object orientation). A spatially compatible combination is considered to activate the associated motor representation (Tucker and Ellis, 1998;
Vainio et al., 2007; Symes et al., 2007; Riggio et al., 2008; see discussion for more information on the nature of the affordance effect). Therefore a higher affordance index would be an indicator for an activated motor representation and thus a facilitated response. We performed two repeated-measures ANOVAs: in the first one we searched for a general affordance effect and used the reaction times as dependent variable, hand, object orientation, and object category as within-subject factors, and group membership as between-subjects factor. In the second ANOVA we looked for differential affordance effects depending on the stimulus category and the group membership, i.e., we used the affordance index as dependent variable, object category as within-subject factor and group membership as between-subjects factor. The individual affordance indices of the smokers for everyday and smoking-related objects were correlated (Pearson correlation) with the corresponding FTND scores.

\section{Results}

\section{fMRI study}

The two-way whole-brain RFX ANOVA with the factors "stimulus category" (smoke vs control) and "group" (smokers vs nonsmokers) revealed a significant stimulus category $\times$ group interaction in the regions listed in Table $1(F \geq 6.1 ; p<0.001$, corrected for multiple comparisons). The ROI-based $t$ tests indicated higher fMRI activation for smokers compared with nonsmokers during the presentation of smoking cues in mainly two sets of brain regions which were identified by the whole-brain RFX ANOVA interaction. The first set consisted of left ventral and dorsal striatum, dorsolateral prefrontal cortex (DLPFC), insula (Fig. 1B), uncus and medial frontal gyrus, right posterior subcallosal gyrus, and bilateral parahippocampal gyrus (PhG). In line with our hypotheses, the second brain network comprised bilateral premotor cortex (PMC) (Fig. 1A), left superior parietal lobule (SPL) (Fig. 1C), and right lateral cerebellum (Fig. 1D).

For the analysis of correlations between the individual degree of nicotine dependence and fMRI cue reactivity, a whole-brain RFX ANCOVA with the contrast "smoke > control" and the covariate "FTND score" was computed ( $n=15$ smokers) (Table 2). The $\beta$ weights of the GLM-contrast "smoke $>$ control" and the individual FTND scores were significantly correlated in left SPL $(r=0.67, p<0.01)$ and a region comprising parts of posterior middle temporal gyrus/inferior temporal cortex/fusiform gyrus (pMTG/ITC/FG; $r=0.70, p<0.01$ ), right premotor cortex 


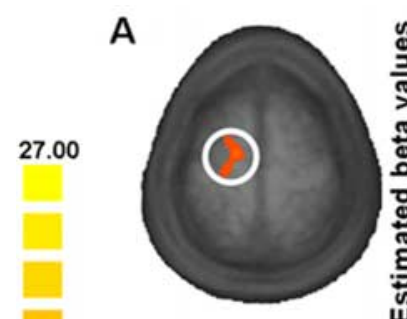

left premotor cortex, $z=61$

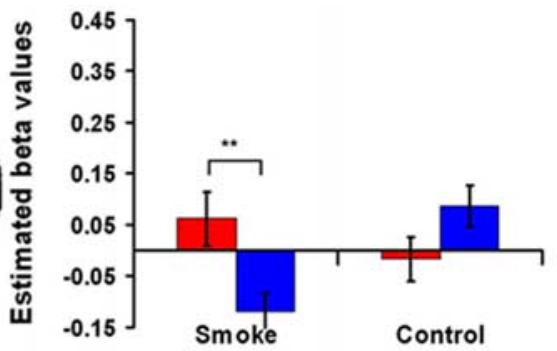

Control
B

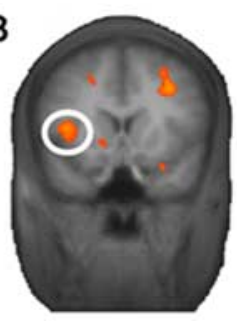

left insula, $y=12$

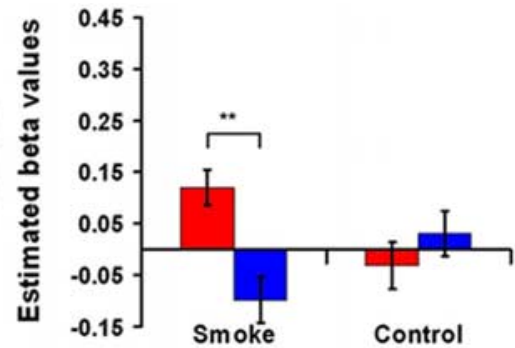

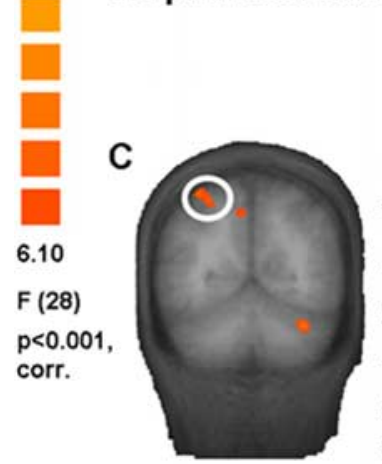
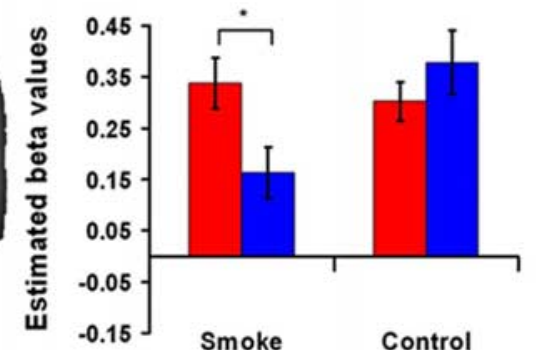

Control

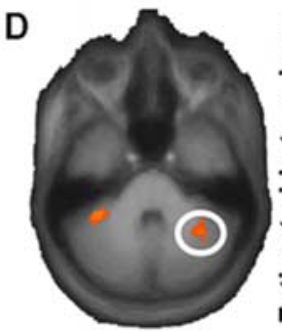

right cerebellum, $z=-32$

left superior parietal lobule, $y=-54$

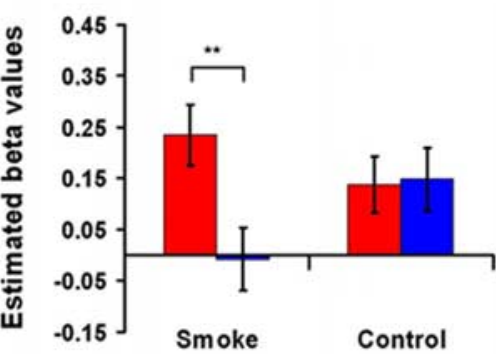

Smoke
Control

Figure 1. Neural cue reactivity in smokers and nonsmokers. A whole-brain RFX ANOVA interaction "stimulus category $\times$ group" revealed mainly two networks of brain regions, where ROI-based $t$ tests showed higher fMRl activations in smokers than nonsmokers while viewing smoking-related images. The first network consisted of regions related to craving and reward motivation (e.g., insula, see $\boldsymbol{B}$ ), and the second one comprised regions associated with skillful tool use and action knowledge (e.g., premotor cortex, superior parietal lobule, and lateral cerebellum; see $\boldsymbol{A}, \boldsymbol{C}$, and $\boldsymbol{D}$, respectively). The scale on the left side indicates minimum and maximum $F$ values, the degrees of freedom, and the significance level for the interaction. Error bars indicate $S E$ from the mean; ${ }^{*} p<$ $0.05 ;{ }^{* *} p<0.01$.

Table 2. Brain regions showing significant results for the whole-brain RFX ANCOVA with contrast"smoke $>$ control" and covariate FTND ( $d f=13, p<0.001$, corrected for multiple comparisons)

\begin{tabular}{lrrrrrr}
\hline & \multicolumn{3}{l}{ Talairach coordinates } & \multirow{2}{*}{ No. of } \\
\cline { 2 - 4 } Region & $x$ & $y$ & $z$ & voxels & $r$ value \\
\hline Left posterior middle temporal gyrus/inferior & & & & & \\
$\quad$ temporal cortex/fusiform gyrus & -40 & -66 & -2 & 4021 & 0.70 \\
Right inferior temporal cortex/fusiform gyrus & 36 & -63 & -8 & 4405 & 0.69 \\
Right premotor cortex & 37 & 3 & 28 & 418 & 0.66 \\
Right middle frontal gyrus & 36 & 22 & 22 & 379 & 0.68 \\
Right middle occipital gyrus & 30 & -79 & 8 & 555 & 0.67 \\
Left superior parietal lobule & -27 & -66 & 52 & 834 & 0.67 \\
\hline
\end{tabular}

$(r=0.66, p<0.01)$, ITC/FG $(r=0.69, p<0.01)$, middle frontal gyrus (MFG; $r=0.68, p<0.01)$, and middle occipital gyrus $(r=$ $0.67, p<0.01$ ) (Fig. 2).

\section{Behavioral study}

There were no main effects or interactions involving the factors object category or group on reaction times or error rates (Fig. $3 A)$. However, we found a hand $\times$ object orientation ANOVA interaction $(p<0.05)$, i.e., a general affordance effect. This interaction was attributable to shorter reaction times for compatibility compared with incompatibility between responding hand and object orientation of the priming visual object. There was a hand $\times$ object orientation $\times$ group $\times$ object category interaction $(p<0.05)$ which indicated that the facilitation of responses compatible to object orientation depended on group membership and object category. To present this specific effect more clearly, we computed "affordance indices" indicating the amplitude of the respective affordance (i.e., facilitation) effect on the basis of the reaction times (see Materials and Methods). The ANOVA with the affordance index as dependent variable revealed a group $\times$ object category interaction $(p<0.05)$ (Fig. $3 B)$. While smokers and nonsmokers showed comparable affordance effects for everyday nonsmoking objects, smoking paraphernalia led to affordance effects in smokers only. Furthermore, in smokers the FTND scores were significantly correlated with the affordance effect for smoking-related objects $(r=0.71, p=0.05)$ (Fig. $3 C$ ) but not for everyday objects $(r=-0.4)$ (Fig. $3 D)$.

The whole-brain RFX ANCOVA with the contrast "smoke > control" and the covariate "affordance index for smoking objects" ( $n=8$ smokers) (Table 3$)$ revealed left PMC $(r=0.90, p<$ $0.01)$, SPL $(r=0.90, p<0.01)$, MFG $(r=0.90, p<0.01)$, and Brodmann area 7 in the parietal lobe $(r=0.90, p<0.01)$ as well as right ITC $(r=0.90, p<0.01)$ and a region in cuneus/precuneus $(r=0.90, p<0.01)$ (Fig. 4$)$.

The behavioral orientation affordance experiment showed an increased behavioral responsiveness (in terms of the orientation affordance effect) to smoking-related objects in smokers than nonsmokers.

\section{ROI-based analyses}

Within the regions revealed by the whole-brain RFX ANCOVA with the contrast "smoke $>$ control" and the covariate "FTND score", a further ROI-based RFX ANCOVA was computed with the contrast "smoke $>$ control" and the covariate "affordance index for smoking objects" ( $n=8$ smokers) (Table 4). Left SPL $(r=0.72, p<0.05)$ and pMTG/ITC/FG $(r=0.74, p<0.05)$ showed significant correlations not only for the whole-brain, but also for the ROI-based RFX ANCOVA. 


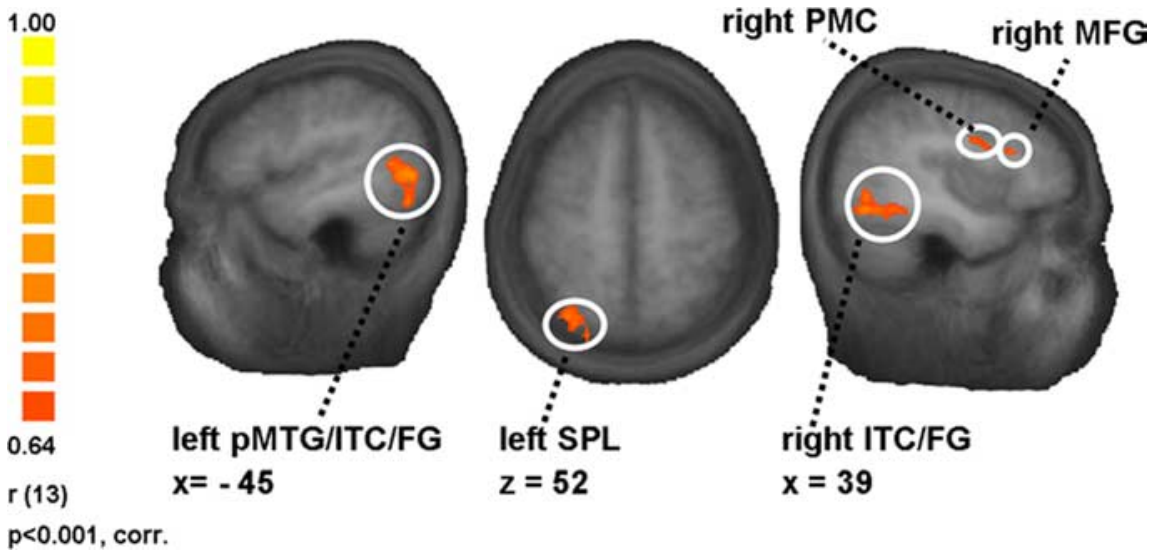

Figure 2. Association between neural cue reactivity in smokers and the individual degree of nicotine dependence. The figure illustrates some of the regions where the covariate "FTND score" correlated significantly with the $\beta$ values of the whole-brain RFX ANCOVA contrast "smoke $>$ control" ( $n=15$ smokers). For a full list of the regions, see Table 2 . The scale on the left side indicates minimum and maximum $r$ values, the degrees of freedom, and the significance level for the whole-brain RFX ANCOVA.

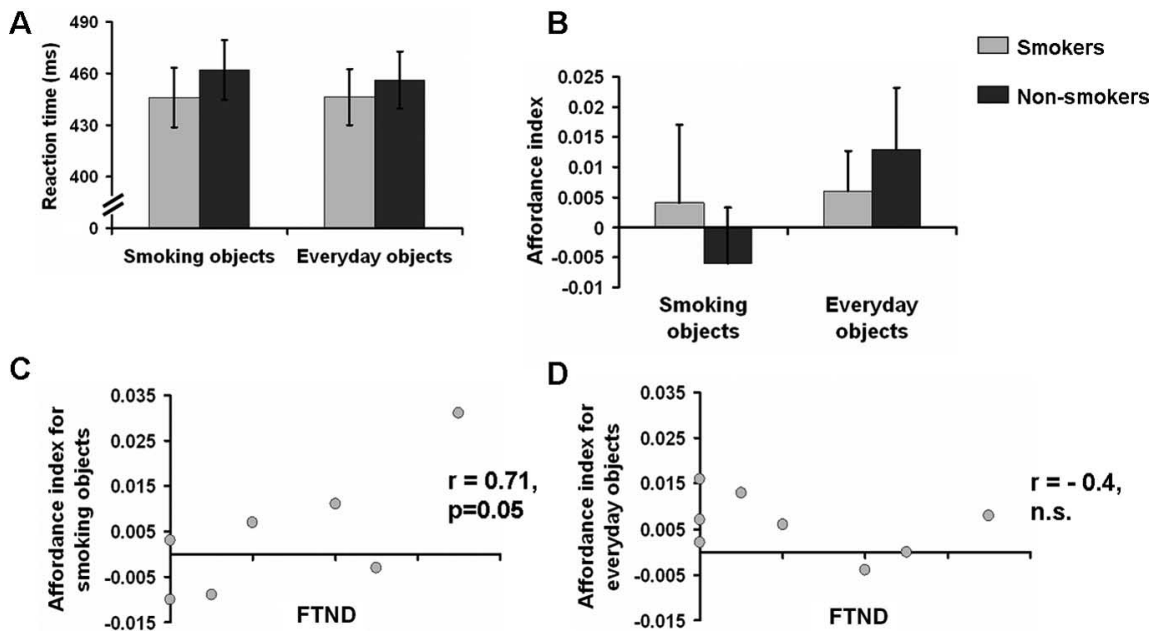

Figure 3. Behavioral cue reactivity of smokers and nonsmokers and association of the automatized responsiveness of smokers to the individual level of nicotine dependence. $A$, Reaction times (means and SEs) of the two groups (smokers vs nonsmokers) for the two object categories (smoking objects vs everyday objects). $\boldsymbol{B}$, Affordance indices (means and SEs) of the two groups (smokers vs nonsmokers) for the two object categories (smoking objects vs everyday objects) (ANOVA interaction, $p<0.05$ ). $\boldsymbol{C}$, Correlation between smokers' affordance indices for smoking objects and FTND scores $(r=0.71, p=0.05 ; n=8$; two of the smokers have identical affordance indices and FTND scores). $\boldsymbol{D}$, Correlation between smokers' affordance indices for everyday objects and FTND values ( $r=-0.4$, n.S.; $n=8)$.

All of the regions revealed by the whole-brain RFX ANCOVA with the contrast "smoke $>$ control" and the covariate "affordance index for smoking objects" with the exception of left BA 7 and right cuneus/precuneus showed significant correlations also in the ROI-based RFX ANCOVA ( $n=8$ smokers, significant correlations varying between 0.76 and 0.80 ) with the contrast "smoke > control" and the covariate "FTND" (Table 5).

\section{Discussion}

Our analyses revealed mainly two networks of brain regions. The first one consisted of regions known to mediate the motivational aspects of addiction, while the second one consisted of brain regions related to tool use knowledge and action representation. Activations of some of the latter regions were correlated with the level of nicotine dependence.

The first network comprised the left ventral and dorsal striatum, DLPFC, insula, uncus, and medial frontal gyrus as well as right posterior subcallosal gyrus and bilateral $\mathrm{PhG}$. These regions have been associated with subjective responses of craving and the reward system (Breiter et al., 1997; Brody et al., 2002; Grüsser et al., 2004; Wilson et al., 2004; Chiamulera, 2005; Naqvi et al., 2007; Fregni et al., 2008). The ventral striatum has been proposed to mediate the reinforcing effects of drugs (Drevets et al., 2001; Kelley and Berridge, 2002; Volkow et al., 2004). The dorsal striatum is known for its role in stimulus-response learning and habitual behaviors (Packard and Knowlton, 2002) and may be of great importance for compulsive drug-taking behavior (Gerdeman et al., 2003; Hyman et al., 2006). Moreover, dorsal striatal dopaminergic activity has been shown to be associated with cue-induced cocaine craving and could thus reflect the automatized nature of craving in later stages of addiction (Volkow et al., 2006). Ventral domains of the striatum may be more important for the early stages of addiction, whereas the more dorsal domains may take over control in the later stages, when drug usage is easily triggered by conditioned stimuli and is executed automatically (Everitt and Robbins, 2005). The higher activations in smokers compared with nonsmokers when exposed to smoking cues may thus reflect reward-related processes or experience of craving. Activations in the dorsal striatum may form the neural basis for the habitual link between conditioned cues and smoking behavior. The fact that smokers exhibited higher BOLD signals in both the ventral and dorsal parts of the striatum can be attributed to the fact that the smokers' group consisted both of light and heavy smokers. Interestingly, we found no correlations between the degree of nicotine dependence and activations in this craving-related network.

The second network comprising bilateral PMC, left SPL, and right lateral cerebellum might underlie the representation of drug-taking skills as well as the preparation and efficient execution of the specific action sequences. These regions are thought to form part of a system representing tool use skills and action knowledge (Grafton et al., 1997; Chao and Martin, 2000; Grèzes et al., 2003; Johnson-Frey, 2004) and to be crucial for the efficient execution of the corresponding actions (Creem-Regehr and Lee, 2005; Lewis, 2006). The premotor cortex is important for linking intent or knowledge of how to execute an action to the following sequence of motor commands as well as for selecting actions on the basis of arbitrary cues (Fink et al., 1999; Arbib et al., 2000; Grèzes et al., 2003). The lateral cerebellum is known to encode skilled action representations, internal models of tools, and automatized actions (Imamizu et al., 2003; Higuchi et al., 2007). The left superior parietal lobule has a prominent role in planning and executing tool use movements (Johnson-Frey et al., 2005). Apparently, smoking cues automatically activate smokers' neural representations of the corresponding tool use skills and action knowledge. 
Table 3. Brain regions showing significant results for the whole-brain RFX ANCOVA with contrast "smoke > control" and covariate "affordance index for smoking objects" ( $\mathrm{df}=6, p<0.001$, corrected for multiple comparisons)

\begin{tabular}{|c|c|c|c|c|c|}
\hline \multirow[b]{2}{*}{ Region } & \multicolumn{3}{|c|}{ Talairach coordinates } & \multirow[b]{2}{*}{ No. of voxels } & \multirow[b]{2}{*}{$r$ value } \\
\hline & $x$ & $y$ & $z$ & & \\
\hline Left premotor cortex & -34 & 2 & 51 & 1653 & 0.90 \\
\hline Left superior parietal lobule & -37 & -66 & 41 & 944 & 0.90 \\
\hline Right inferior temporal cortex & 44 & -59 & -5 & 649 & 0.90 \\
\hline Left middle frontal gyrus & -45 & 35 & 14 & 588 & 0.90 \\
\hline Left parietal lobe, BA 7 & -10 & -51 & 36 & 529 & 0.90 \\
\hline Right cuneus/precuneus & -28 & -70 & 15 & 527 & 0.90 \\
\hline
\end{tabular}

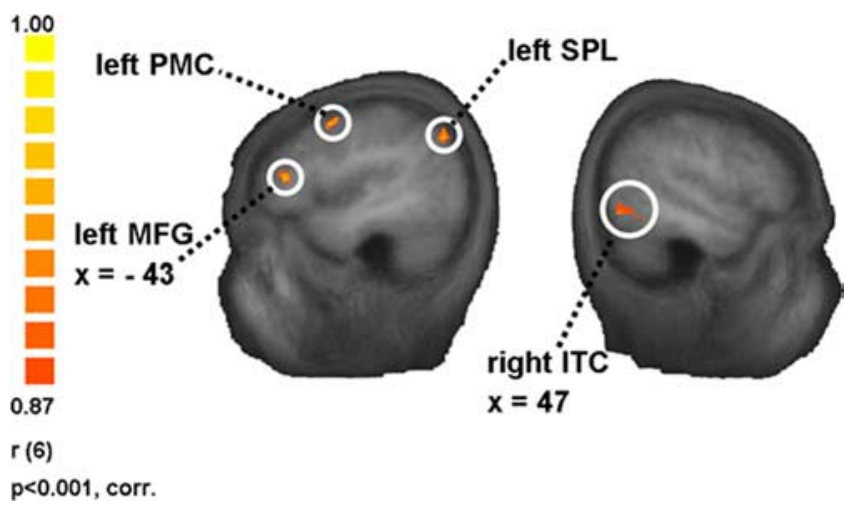

Figure 4. Association between neural cue reactivity in smokers and automatized behavioral responsiveness to smoking cues. The figure illustrates some of the regions where the covariate "affordance index for smoking objects" was significantly correlated with the $\beta$ values of the whole-brain RFX ANCOVA contrast "smoke $>$ control" ( $n=8$ smokers). For a full list of the regions, see Table 3 . The scale on the left side indicates minimum and maximum $r$ values, the degrees of freedom, and the significance level for the whole-brain RFX ANCOVA.

The association between the individual level of nicotine dependence and the $\beta$ values from the whole-brain RFX ANCOVA in left SPL, left pMTG/ITC/FG, and right PMC and MFG further supports the assumed relationship between the neural representations of drug-related action schemata which are dependent on the smoker's learning history (Tiffany, 1990), and the severity of nicotine dependence. The FG/ITC is related both to the analysis of object features and to the interpretation of visual action (Haxby et al., 2001; Chaminade et al., 2005). The pMTG is involved in processing action knowledge (Johnson-Frey et al., 2005). Several studies have pointed out the importance of MFG for skilled movement and tool-associated action identification (Grabowski et al., 1998; Haaland et al., 2000). Increased smoking expertise may thus include an enhanced ability to activate the respective smoking skill and action representations. Behavioral evidence also suggests that habitual, automatized actions are a frequent reason for smoking in more dependent smokers (Piasecki et al., 2007).

The absence of a correlation between FTND score and fMRI activation in craving-related brain regions may be attributable to the fact that our participants were not nicotine-deprived. Indeed, there is evidence that nicotine deprivation and cigarette availability affect cue reactivity (Droungas et al., 1995; Carter and Tiffany, 2001; McBride et al., 2006). However, our aim was to investigate reactivity as closely as possible to natural everyday smoking situations. Furthermore, it has been shown that craving occurs also in natural environments without an experimentally induced nicotine deprivation (Piasecki et al., 2007).

Our second experiment investigated the orientation affordance effect as a function of group and object category. Orientation affordance refers to faster responses for spatial compatibility between the responding hand and the orientation of the priming image of a graspable object. It has been proposed that this facilitation of reactions is attributable to the automatic activation of corresponding motor representations by the priming object (Tucker and Ellis, 1998; Symes et al., 2007; Vainio et al., 2007; Riggio et al., 2008). Thus, the affordance effect represents a suitable measure of automatized smoking-related action representations. Our data provide evidence for a general affordance effect, indicating faster reactions for congruency between the responding hand and the orientation of the priming object. Smokers and nonsmokers exhibited comparable affordance effects for common nonsmoking objects. Smoking paraphernalia led to affordance effects in smokers only, i.e., reactions to smoking-related objects were facilitated by compatible object orientation in smokers but not in nonsmokers. Thus, smokers' responses to smoking paraphernalia can be triggered as automatically and easily as to comparable everyday objects. Furthermore, experience-based motor representations of smoking actions have been established in smokers only.

Although there was a general affordance effect and a "group $X$ object category" interaction for the affordance index, there were no general reaction time differences between the groups and no "group $\times$ object category" interaction for the reaction times. This was not unexpected since only the affordance index takes the compatibility between responding hand and object orientation into account and measures the action facilitation effect.

The FTND scores correlated with the affordance effect of smokers for smoking but not for everyday objects. Furthermore, the whole-brain RFX ANCOVA revealed remarkable correlations between the covariate "affordance index for smoking objects" and fMRI cue reactivity in left PMC, SPL, MFG, and right ITC. This shows that automatized behavioral responsiveness to smoking-associated cues is more pronounced in heavier smokers and is related to the neural underpinnings of drug-taking skills. Thus, the smokers' experience with smoking-related objects was reflected in both behavioral affordance profiles and the activation of tool use-related brain regions.

The fMRI cue reactivity in the majority of those regions which were identified by the whole-brain RFX ANCOVAs using the one covariate also showed a significant ROI-based correlation with the other covariate. This further supports the association of fMRI activation of action-related brain regions with the severity of nicotine dependence and automatized behavioral responsiveness to smoking-related objects.

The strong activation of smoking-related action schemata in smokers might either be interpreted as a cause of smoking or as an effect of exaggerated consumption. Research on experiencebased neural plasticity shows that intensive engagement in an activity leads to stronger neural representations of the respective activity and the used objects (Pearce et al., 2000; Sanes and Donoghue, 2000; Calvo-Merino et al., 2006; Schwenkreis et al., 2007; 
Table 4. ROI-based RFX ANCOVA with the contrast "smoke > control" and the covariate "affordance index for smoking objects" (df $=6)$ was computed within brain regions showing significant results for the whole-brain RFX ANCOVA with contrast"smoke > control" and covariate FTND

\begin{tabular}{|c|c|c|c|c|c|}
\hline \multirow[b]{2}{*}{ Region } & \multicolumn{3}{|c|}{ Talairach coordinates } & \multirow[b]{2}{*}{ No. of voxels } & \multirow[b]{2}{*}{$r$ value (ROI-based RFX ANCOVA) } \\
\hline & $x$ & $y$ & $z$ & & \\
\hline \multicolumn{6}{|l|}{ Left posterior middle temporal gyrus/inferior } \\
\hline temporal cortex/fusiform gyrus & -40 & -66 & -2 & 4021 & $0.74^{*}$ \\
\hline Left superior parietal lobule & -27 & -66 & 52 & 834 & $0.72^{*}$ \\
\hline Right premotor cortex & 37 & 3 & 28 & 418 & 0.67, n.s. \\
\hline Right middle occipital gyrus & 30 & -79 & 8 & 555 & 0.54, n.s. \\
\hline Right inferior temporal cortex/fusiform gyrus & 36 & -63 & -8 & 4405 & 0.49, n.s. \\
\hline Right middle frontal gyrus & 36 & 22 & 22 & 379 & 0.31 , n.s. \\
\hline
\end{tabular}

${ }^{*} p<0.05 ;$ n.s., not significant.

Table 5. ROI-based RFX ANCOVA with the contrast"smoke > control" and the covariate"FTND" (df = 6) was computed within brain regions showing significant results for the whole-brain RFX ANCOVA with contrast "smoke > control" and covariate "affordance index for smoking objects"

\begin{tabular}{|c|c|c|c|c|c|}
\hline \multirow[b]{2}{*}{ Region } & \multicolumn{3}{|c|}{ Talairach coordinates } & \multirow[b]{2}{*}{ No. of voxels } & \multirow[b]{2}{*}{$r$ value (ROI-based RFX ANCOVA) } \\
\hline & $x$ & $y$ & $z$ & & \\
\hline Left superior parietal lobule & -37 & -66 & 41 & 944 & $0.80^{*}$ \\
\hline Left premotor cortex & -34 & 2 & 51 & 1653 & $0.79 *$ \\
\hline Right inferior temporal cortex & 44 & -59 & -5 & 649 & $0.77^{*}$ \\
\hline Left middle frontal gyrus & -45 & 35 & 14 & 588 & $0.76^{*}$ \\
\hline Right cuneus/precuneus & -28 & -70 & 15 & 527 & 0.63, n.s. \\
\hline Left parietal lobe, BA 7 & -10 & -51 & 36 & 529 & 0.55, n.s. \\
\hline
\end{tabular}

${ }^{*} p<0.05 ;$ n.s., not significant.

Weisberg et al., 2007). Therefore, we suggest that strong activation of the smoking-related action schemata is the consequence of the frequently executed process of smoking. On the other hand, triggered schemata may also reduce the threshold for executing the action of smoking, thus contributing to the high smoking relapse rates.

Together, our findings confirm that nicotine dependence involves striatum, insula, and dorsolateral prefrontal cortex, which have been intensively studied in relation to craving and drugseeking habits. The present results provide new evidence that smoking-associated images can activate neural representations in brain regions probably encoding the respective drug-taking skills and action knowledge. Responses of action-related regions to smoking cues may thus represent a novel neural correlate of nicotine dependence. On the behavioral level, smoking paraphernalia activate motor representations in smokers but not in nonsmokers as easily and automatically as similarly familiar objects. This effect is linked to the fMRI cue reactivity of smokers in tool use and action-related brain regions. Both neural and behavioral correlates of the drug-taking skills and action knowledge correlate with the level of nicotine dependence. The activation of such deeply embedded action schemata might contribute to the high relapse risk in nicotine addiction. We hope that these findings will initiate the development of novel neural and behavioral markers of therapy success or risk of relapse, and guide new therapeutic strategies addressing the importance of involuntary, automatic action sequences.

\section{References}

Arbib MA, Billard A, Iacoboni M, Oztop E (2000) Synthetic brain imaging: grasping, mirror neurons and imitation. Neural Netw 13:975-997.

Baxter BW, Hinson RE (2001) Is smoking automatic? Demands of smoking behavior on attentional resources. J Abnorm Psychol 110:59-66.

Bleich S, Havemann-Reinecke U, Kornhuber J (2002) : Fagerström-Test für Nikotinabhängigkeit (FTNA). Göttingen: Beltz Test.

Breiter HC, Gollub RL, Weisskoff RM, Kennedy DN, Makris N, Berke JD, Goodman JM, Kantor HL, Gastfriend DR, Riorden JP, Mathew RT, Rosen BR, Hyman SE (1997) Acute effects of cocaine on human brain activity and emotion. Neuron 19:591-611.
Brody AL, Mandelkern MA, London ED, Childress AR, Lee GS, Bota RG, Ho ML, Saxena S, Baxter LR Jr, Madsen D, Jarvik ME (2002) Brain metabolic changes during cigarette craving. Arch Gen Psychiatry 59:1162-1172.

Calvo-Merino B, Grèzes J, Glaser DE, Passingham RE, Haggard P (2006) Seeing or doing? Influence of visual and motor familiarity in action observation. Curr Biol 16:1905-1910.

Carter BL, Tiffany ST (2001) The cue-availability paradigm: the effects of cigarette availability on cue reactivity in smokers. Exp Clin Psychopharmacol 9:183-190.

Chaminade T, Meltzoff AN, Decety J (2005) An fMRI study of imitation: action representation and body schema. Neuropsychologia 43:115-127.

Chao LL, Martin A (2000) Representation of manipulable man-made objects in the dorsal stream. Neuroimage 12:478-484.

Chiamulera C (2005) Cue reactivity in nicotine and tobacco dependence: a "multiple-action" model of nicotine as a primary reinforcement and as an enhancer of the effects of smoking-associated stimuli. Brain Res Brain Res Rev 48:74-97.

Creem-Regehr SH, Lee JN (2005) Neural representations of graspable objects: are tools special? Brain Res Cogn Brain Res 22:457-469.

Drevets WC, Gautier C, Price JC, Kupfer DJ, Kinahan PE, Grace AA, Price JL, Mathis CA (2001) Amphetamine-induced dopamine release in human ventral striatum correlates with euphoria. Biol Psychiatry 49:81-96.

Droungas A, Ehrman RN, Childress AR, O’Brien CP (1995) Effect of smoking cues and cigarette availability on craving and smoking behavior. Addict Behav 20:657-673.

Everitt BJ, Robbins TW (2005) Neural systems of reinforcement for drug addiction: from actions to habits to compulsion. Nat Neurosci 8:1481-1489.

Field M, Mogg K, Bradley BP (2006) Automaticity of smoking behaviour: the relationship between dual-task performance, daily cigarette intake and subjective nicotine effects. J Psychopharmacol 20:799-805.

Fink GR, Marshall JC, Halligan PW, Frith CD, Driver J, Frackowiak RS, Dolan RJ (1999) The neural consequences of conflict between intention and the senses. Brain 122:497-512.

Fregni F, Liguori P, Fecteau S, Nitsche MA, Pascual-Leone A, Boggio PS (2008) Cortical stimulation of the prefrontal cortex with transcranial direct current stimulation reduces cue-provoked smoking craving: a randomized, sham-controlled study. J Clin Psychiatry 69:32-40.

Gerdeman GL, Partridge JG, Lupica CR, Lovinger DM (2003) It could be habit forming: drugs of abuse and striatal synaptic plasticity. Trends Neurosci 26:184-192.

Gilbert DG, Rabinovich NE (2006) International smoking image series 
(with neutral counterparts). Carbondale, IL: Integrative Neuroscience Laboratory, Department of Psychology, Southern Illinois University.

Grabowski TJ, Damasio H, Damasio AR (1998) Premotor and prefrontal correlates of category-related lexical retrieval. Neuroimage 7:232-243.

Grafton ST, Fadiga L, Arbib MA, Rizzolatti G (1997) Premotor cortex activation during observation and naming of familiar tools. Neuroimage 6:231-236.

Grèzes J, Tucker M, Armony J, Ellis R, Passingham RE (2003) Objects automatically potentiate action: an fMRI study of implicit processing. Eur J Neurosci 17:2735-2740.

Grüsser SM, Wrase J, Klein S, Hermann D, Smolka MN, Ruf M, Weber-Fahr W, Flor H, Mann K, Braus DF, Heinz A (2004) Cue-induced activation of the striatum and medial prefrontal cortex is associated with subsequent relapse in abstinent alcoholics. Psychopharmacology (Berl) 175:296-302.

Haaland KY, Harrington DL, Knight RT (2000) Neural representations of skilled movement. Brain 123:2306-2313.

Haxby JV, Gobbini MI, Furey ML, Ishai A, Schouten JL, Pietrini P (2001) Distributed and overlapping representations of faces and objects in ventral temporal cortex. Science 293:2425-2430.

Higuchi S, Imamizu H, Kawato M (2007) Cerebellar activity evoked by common tool-use execution and imagery tasks: an fMRI study. Cortex 43:350-358.

Hyman SE, Malenka RC, Nestler EJ (2006) Neural mechanisms of addiction: the role of reward-related learning and memory. Annu Rev Neurosci 29:565-598.

Imamizu H, Kuroda T, Miyauchi S, Yoshioka T, Kawato M (2003) Modular organization of internal models of tools in the human cerebellum. Proc Natl Acad Sci U S A 100:5461-5466.

Johnson-Frey SH (2004) The neural bases of complex tool use in humans. Trends Cogn Sci 8:71-78.

Johnson-Frey SH, Newman-Norlund R, Grafton ST (2005) A distributed left hemisphere network active during planning of everyday tool use skills. Cereb Cortex 15:681-695.

Kelley AE, Berridge KC (2002) The neuroscience of natural rewards: relevance to addictive drugs. J Neurosci 22:3306-3311.

Lewis JW (2006) Cortical networks related to human use of tools. Neuroscientist 12:211-231.

McBride D, Barrett SP, Kelly JT, Aw A, Dagher A (2006) Effects of expectancy and abstinence on the neural response to smoking cues in cigarette smokers: an fMRI study. Neuropsychopharmacology 31:2728-2738.

Miller NS, Gold MS (1994) Dissociation of "conscious desire" (craving) from and relapse in alcohol and cocaine dependence. Ann Clin Psychiatry 6:99-106.

Naqvi NH, Rudrauf D, Damasio H, Bechara A (2007) Damage to the insula disrupts addiction to cigarette smoking. Science 315:531-534.

Packard MG, Knowlton BJ (2002) Learning and memory functions of the basal ganglia. Annu Rev Neurosci 25:563-593.

Pearce AJ, Thickbroom GW, Byrnes ML, Mastaglia FL (2000) Functional reorganisation of the corticomotor projection to the hand in skilled racquet players. Exp Brain Res 130:238-243.

Piasecki TM (2006) Relapse to smoking. Clin Psychol Rev 26:196-215.

Piasecki TM, Richardson AE, Smith SM (2007) Self-monitored motives for smoking among college students. Psychol Addict Behav 21:328-337.

Riggio L, Iani C, Gherri E, Benatti F, Rubichi S, Nicoletti R (2008) The role of attention in the occurrence of the affordance effect. Acta Psychol (Amst) 127:449-458.

Sanes JN, Donoghue JP (2000) Plasticity and primary motor cortex. Annu Rev Neurosci 23:393-415.

Schwenkreis P, El Tom S, Ragert P, Pleger B, Tegenthoff M, Dinse HR (2007) Assessment of sensorimotor cortical representation asymmetries and motor skills in violin players. Eur J Neurosci 26:3291-3302.

Symes E, Ellis R, Tucker M (2007) Visual object affordances: object orientation. Acta Psychol (Amst) 124:238-255.

Tiffany ST (1990) A cognitive model of drug urges and drug-use behavior: role of automatic and nonautomatic processes. Psychol Rev 97:147-168.

Tucker M, Ellis R (1998) On the relations between seen objects and components of potential actions. J Exp Psychol Hum Percept Perform 24:830-846.

Vainio L, Ellis R, Tucker M (2007) The role of visual attention in action priming. Q J Exp Psychol (Colchester) 60:241-261.

Volkow ND, Fowler JS, Wang GJ (2004) The addicted human brain viewed in the light of imaging studies: brain circuits and treatment strategies. Neuropharmacology 47 [Suppl 1]:3-13.

Volkow ND, Wang GJ, Telang F, Fowler JS, Logan J, Childress AR, Jayne M, Ma Y, Wong C (2006) Cocaine cues and dopamine in dorsal striatum: mechanism of craving in cocaine addiction. J Neurosci 26:6583-6588.

Weisberg J, van Turennout M, Martin A (2007) A neural system for learning about object function. Cereb Cortex 17:513-521.

Wilson SJ, Sayette MA, Fiez JA (2004) Prefrontal responses to drug cues: a neurocognitive analysis. Nat Neurosci 7:211-214.

World Health Organization (2008) WHO report on the global tobacco epidemic, 2008: the MPOWER package. Geneva: World Health Organization. 